\title{
Lecciones de humanidad: la importancia del apoyo a familias con hijos hospitalizados por cáncer
}

\section{Lessons from humanity: the importance of supporting families with children hospitalized for cancer}

Ken Eduardo Ávila Roura

Dora Adolfina Ayora Talavera

Teresita Concepción Campo Marín

Universidad Autónoma de Yucatán

\section{Resumen}

Este escrito es resultado de una investigación relacionada con las redes sociales en apoyo a las familias con hijos hospitalizados por cáncer; destacan los tipos de redes que surgen, así como la diversidad de apoyos que brindan y el impacto de la ayuda en el proceso de la enfermedad desde el diagnóstico hasta la recuperación o fallecimiento de los menores. También es una muestra de la capacidad humana de ser sensibles, generosos y proactivos en beneficio de los demás. Se presentan las experiencias de cuatro familias, de las cuales dos pasaron por la muerte del niño y las otras dos se encontraban en tratamiento desde hace, por lo menos, un año. El estudio que se realizó es de corte cualitativo con metodología fenomenológica, ampliando desde la perspectiva de la poesía social, para recuperar el discurso de las familias, así como el proceso de los investigadores al ser testigos externos de las historias.

Palabras clave: cáncer, familia, redes sociales, poesía social, humanidad.

Nota del autor

Ken Eduardo Ávila Roura, Universidad Autónoma de Yucatán. Dora Adolfina Ayora Talavera, Universidad Autónoma de Yucatán (UADY), Carr. Mérida-Tizimín, km 1, Cholul, Yucatán, C. P. 97305. Celular: 9999475335, correo electrónico: ayotalav@correo.uady.mx Teresita Concepción Campo Marín, Universidad Autónoma de Yucatán. 


\begin{abstract}
The current manuscript provides evidence of the importance of social networks in families with hospitalized children. It emphasizes the role of networks and their impact on family support. Additionally, it provides an example of the human capacity to be sensitive, generous and proactive for others. The sample included four families, two of which experienced the loss of their child and the other two had their children in treatment for at least a year. The study used qualitative methods with a phenomenological approach. A social poetry perspective as well as researchers as external witnesses of the stories process were used to gather family discourses.
\end{abstract}

Keywords: cancer, family, social networks, social poetry, humanity.

Las consecuencias que genera una enfermedad crónica como el cáncer, impactan la calidad de vida, sobre todo en lo que se refiere a la salud física, emocional y social de los diferentes miembros de una familia. Cuando la persona diagnosticada es un menor, los cuidados recaen sobre los padres, quienes deben afrontar toda la carga emocional, además de tomar decisiones relativas a qué hacer ante imprevistos o necesidades emergentes (esto desde el diagnóstico), cómo organizarse para visitas hospitalarias y cuidados del seguimiento del tratamiento, quién será el cuidador primario y quiénes fungirán como secundarios, y qué adecuaciones harán al presupuesto familiar. Las decisiones que vayan tomando tendrán repercusión en las diferentes áreas de su vida y derivarán en cambios en la rutina cotidiana, que les permitirán organizarse y afrontar los retos de la enfermedad en diversos contextos como el trabajo, la escuela y la propia casa (López-Huerta, Álvarez-Bermúdez, \& González-Romo, 2012; Eiser, Eiser, \& Stride, 2005; Grau, \& Espada, 2012). También surge una serie de situaciones para la cual los miem- bros no tienen una preparación previa, a saber, relacionarse con el personal médico, el traslado a lugares nuevos (como hospitales y consultorios, en ocasiones a una ciudad distinta a la suya), manejo de los cuidados médicos en casa, la separación de familiares y amigos, así como cambios en el rol social (López-Huerta et al., 2012; Eiser, Eiser, \& Stride, 2005).

De manera similar, Ávila (2016) menciona en su estudio, que es evidente el gran impacto que tiene el proceso de la enfermedad en la familia, desde el momento del diagnóstico, ya que pone en juego factores antes ajenos a la familia como los tratamientos, las hospitalizaciones, los trámites burocráticos, los procedimientos quirúrgicos, los altibajos en la salud, entre muchos otros (p. 14). Dada la complejidad de situaciones que las familias deben afrontar, la participación de otras personas se vuelve indispensable; contar con diferentes redes de apoyo brinda oportunidades para que las familias puedan lograr atender todas las demandas que el proceso de enfermedad les impone. 
Es tal la importancia de las redes sociales que han surgido múltiples investigaciones que exploran desde diversas perspectivas el papel que éstas juegan en apoyo a las familias. Algunos autores se centran en el estudio de los diversos tipos de apoyo (García, 2005) y otros se vuelcan hacia las repercusiones en la salud mental, emociones, sentido de existencia y formas de aprontamiento (García, 2005; Barreto, \& Saiz, 2007; Rosemblatt, 1999; García, \& Day, 2009; Castañeda, 2006; Frankl, 1973).

García (2005) en su estudio destaca que las redes sociales son factores de prevención a situaciones críticas, tanto para el afrontamiento de una enfermedad crónica, como para la misma hospitalización. Dentro de las principales fuentes de apoyo se encuentra la familia nuclear, los profesionales de la salud, otras familias de niños hospitalizados y grupos religiosos; el tipo de apoyo recibido consta de apoyo moral, financiero y material; en algunas ocasiones, se llega a recibir apoyo de amigos y vecinos.

Por su parte, Barreto y Siz (2007) reportan que tener familiares y amigos con los que los familiares puedan desahogarse verbalmente produce un mejoramiento en la salud mental, evitando el factor de riesgo del aislamiento. Rosemblatt (1999) estudia el apoyo social como una herramienta útil que aporta distracción a los familiares y los ayuda a salir de la tristeza, estimula emociones positivas que surgen al estar en contacto con otros y al recibir consuelo de las mismas.
Por su parte, García y Day (2009) refieren que las redes son esenciales dentro de la cotidianeidad de los individuos, a tal grado que, quienes carecen de éstas, tienden a tener una vulnerabilidad mayor y presentan efectos adversos relacionados con cuestiones estresantes; de igual manera, Castañeda (2006) ha encontrado que existe una relación inversamente proporcional entre la desesperanza y el apoyo social. Frankl (1973) reporta que a pesar de la brevedad que pudieran tener las interacciones sociales, llegan a brindar apoyo, el acompañar a una persona que pasa por un momento adverso puede llevar a encontrar sentido a la existencia.

Aunque existen líneas de aproximación al papel de las redes sociales en este tipo de contextos, éstas pueden mirarse desde otras perspectivas que profundicen y resalten el valor de lo humano, de las relaciones entre las familias y las redes sociales en situaciones críticas como las vinculadas con cáncer. Este articulo quiere posicionarse y mostrar otra perspectiva, donde los autores nos situamos dentro del proceso de investigación no solo para dar voz a los entrevistados, sino para incorporar una lectura desde nuestra interpretación, lo cual se vincula con lo que se conoce como poesía social: enfatiza la importancia de los seres humanos que participan en las relaciones y la construcción del mundo a partir de ellas.

McNamee (2013) entiende la poesía social como la posibilidad de investigar aquello nuevo que surge en la relación entre las personas, pri- 
vilegiando el compromiso con ellas, permitiendo la creación de nuevas realidades, al entender que los involucrados colaboran en la formación de juicios, valores y procesos de decisión relacionales, construyendo nuevos significados.

Para Deissler y McNamee (2013), la poesía social se ve reflejada en la terapia cuando afirma para crear momentos poéticos es importante generar una estimulación psicoterapéutica, para lo cual el primer paso es estar dispuesto a dejarse impresionar por las palabras (...) una historia emocionante como parte de una conversación, atraerá casi seguro la atención del oyente y lo conmoverá. De ahí que puede afirmarse que de estas conversaciones se sale distinto de cómo se entra (p. 19).

Así como en la terapia, en esta investigación los momentos poéticos pueden verse reflejados en el compartir de las experiencias de las familias y sus redes sociales. La poesía social rescata los aspectos humanos de las relaciones, para mostrarlas como algo más allá de categorías y mirar el valor emocional de las experiencias expresadas o compartidas por las familias participantes.

\section{Método}

\section{Objetivo}

Analizar las experiencias de las familias que tienen un hijo con cáncer en su relación con las redes de apoyo a partir de la perspectiva de la poesía social, para ofrecer una reflexión alternativa a la manera tradicional como han sido vistas las redes de apoyo.

\section{El tipo de estudio}

El estudio que se realizó es de corte cualitativo con metodología fenomenológica, la cual de acuerdo a Vieytes (2009), tiene como objetivo "el campo de los fenómenos tal como son vividos y experimentados por los individuos", esto, ampliado desde la perspectiva de la poesía social, recupera el discurso emitido por las familias, así como también, rescata el proceso de los investigadores al ser testigos externos de las historias. En otras palabras, es un estudio donde se construye la realidad, desde el marco de referencia de los participantes: familias e investigadores.

\section{Participantes}

Los participantes fueron cuatro familias con un menor con diagnóstico de cáncer (dos niñas y dos niños), entre los 4 y 8 años de edad, que recibían tratamiento en el pabellón de Pediatría de la Unidad Médica de Alta Especialidad del Centro Médico Nacional "Ignacio García Téllez" T1 y cuya participación fue voluntaria, dada la cercanía y la confianza que ya existía con uno de los autores, a quien conocieron a lo largo del tratamiento en el que se encontraban por lo menos desde año antes del estudio. Cabe resaltar que dos de las familias enfrentaron el fallecimiento de los menores, una antes del estudio y una más durante el proceso de éste. A continuación, presentamos una breve descripción de la composición de las familias.

La familia de Andrés está conformada por cinco miembros: la madre, Alma, que se dedicaba 
a cuidar de su hogar, y es la proveedora a través de diversos oficios, principalmente limpiando casas particulares; la hermana mayor, Mariela, ya no vive con ellos, se casó y cuida a sus hijos; Adriana, de 14 años, estudia la secundaria; Mariana, de 12 años, cursa la primaria y, por último, Andrés, quien fue diagnosticado cuando tenía 3 años y medio.

La segunda familia fue la de Pedro, consta de seis miembros: madre, Esmeralda, de aproximadamente 32 años, trabaja en un supermercado como cajera; Jesús, el padre, de aproximadamente 30 años, trabaja como mesero y sus turnos rotan. La abuela, Bruna, y el abuelo, Homero, se dedican al cuidado del hogar, aunque don Homero en ocasiones regresa al pueblo de donde es originario; Pedro de 7 años y Vanesa de 3 años.

La familia de Sabina está conformada por su padre, Eusebio de 43 años, repartidor en una farmacia; la madre, Karen, con quien no se tuvo contacto, ya que no pudo participar en las entrevistas debido a su horario de trabajo, los abuelos Joaquina y Miguel.

Por último, la familia de Sofía, formada por Arcelia, la madre, quien se dedicaba al cuidado del hogar y de los hijos; Maximiliano, el padre, proveedor de la casa; Felipe, de 3 años; Alicia de 6 años y Sofía, de 8 años, ambas estudiantes.

Por otra parte, los investigadores pertenecemos a la Facultad de Psicología de la UADY. Ken es autor principal de la tesis de la que deriva este artículo, Dora fue asesora de la tesis y junto con Concepción, los tres revisamos y reanalizamos las narrativas de las familias.

\section{Técnicas}

\section{Recolección de datos}

En cuanto a las técnicas, se realizaron entrevistas semiestructuradas para conocer diferentes aspectos de las experiencias. Para Dezin (1978, citado en Ruiz-Olabuénaga, 2007), la entrevista semiestructurada es una conversación en la que se ejercita el arte de preguntar y escuchar. La entrevista comprende el desarrollo de una interacción entre los participantes (familias e investigadores), en la que influyen las características personales (biológicas, culturales, sociales, conductuales) de los mismos, aunque toma como base un guion, éste puede irse adaptando y modificando según el desarrollo de la conversación.

De acuerdo a Deissler y McNamee (2013), con estas conversaciones, se busca entender, re-crear, transformar y gobernar las realidades. Partiendo de la concepción de que no vemos al mundo a través de ópticas biológicas, fisiológicas y psicológicas, sino que entendemos y formamos nuestro mundo de relaciones, hablando unos con otros.

Vinculado a la poesía social, entendemos la entrevista como una forma de conversación cuya variedad de formas es quizás tan interminable como las posibilidades de comunicación humana. Al igual que en la terapia y retomando a Deissler y McNamee (2013), si uno toma la determinación de ejercer la profesión de 
psicoterapeuta-investigador, toma por ende la decisión de ver el mundo como diálogos y describirse a sí mismo como parte integrante de éstos. Entrar en contacto con las familias $\mathrm{y}$ pedirles que compartan sus experiencias, es ante todo, el requerimiento de que narre una historia que ponga de manifiesto cómo el narrador o narradora describe su problema, qué relación guarda éste con otras personas y qué posibilidades de desarrollo ven.

Para entablar este diálogo, se tomaron como guía de las entrevistas catorce preguntas orientadas a los motivos de hospitalización, impacto del proceso de enfermedad en la familia, principales cambios y ajustes, dificultades y problemas enfrentados, retos de las recaídas, así como adaptaciones después del fallecimiento. Estos temas se exploraron a profundidad en el lapso de tres o cuatro entrevistas con duración aproximada de 50 minutos cada una.

\section{Análisis de datos}

El análisis de contenido es la técnica que se empleó para el análisis, interpretación y compresión de las experiencias recuperadas de las entrevistas. Krippendorff (1990), la define con una técnica de investigación destinada a formular inferencias reproducibles y válidas que pueden aplicarse a su contexto, desde un marco de referencia que abarque: los datos tal como son comunicados, el contexto, la forma en que el conocimiento del investigador obliga a dividir su realidad, el objetivo del análisis, la inferencia como tarea intelectual básica y la validez como criterio supremo de éxito.
Dado el carácter cualitativo, el análisis de la información se vuelve un proceso dinámico y creativo que se alimenta de la experiencia directa del investigador en el escenario estudiado (Amezcua, 2000).

El análisis de contenido, como una forma de entendimiento, es equiparable a la conversación terapéutica, que de acuerdo a Gergen y Gergen (2013), es una forma de dar significado, como un proceso de coordinación humana, a través del cual se construyen y reconstruyen el pasado, el presente y el futuro. La esperanza y la promesa de este proceso es que, a partir de la vertiginosa concatenación de los significados, cada experiencia compartida tome de alguna manera una nueva significación.

Es así que desde el análisis de contenido se puede rescatar la dimensión poética del lenguaje (Gergen, \& Gergen, 2013) que nos permita trascender las interpretaciones convencionales que tienden a la simple categorización de las experiencias cotidianas, y lograr de ese modo alcanzar el sentido de lo humano.

\section{Procedimiento}

Se hizo una invitación abierta a familias que participaron en el proyecto de servicio social "Caza Sonrisas” (Ávila, 2016) y a las referidas por la doctora encargada del pabellón de Pediatría del hospital. Considerando que estuvieran en tratamiento al menos por un año $y$ que residieran en Mérida.

Cuatro familias aceptaron voluntariamente la invitación, dada la cercanía que existía con uno de los investigadores vinculado al proyecto Caza 
Sonrisas. Previa cita, él realizó las entrevistas, con tres de las familias se llevaron a cabo en sus hogares, y con la cuarta, solo se hizo una en la sala de espera del hospital, dado que la niña falleció antes de programar la siguiente. Las entrevistas fueron grabadas y a partir de la transcripción se realizó el análisis de contenido;

\section{Consideraciones éticas}

Toda la investigación se apegó a las normas éticas mencionadas por el Reglamento de la Ley General de Salud en Materia de Investigación para la Salud en su Título Segundo, en el artículo 13 que indica que ha de prevalecer el criterio del respeto a su dignidad y la protección de sus derechos y bienestar. Por lo que todos los nombres fueron cambiados para proteger la identidad de las familias participantes, las cuales firmaron un consentimiento informado y autorizaron la grabación de las entrevistas, garantizando su uso con fines académicos.

\section{Resultados}

Los resultados de este estudio muestran clara concordancia con los estudios tradicionales sobre las redes sociales (García, 2005; Barreto, \& Saiz, 2007; Rosemblatt, 1999; García, \& Day, 2009; Castañeda, 2006; Frankl, 1973; LópezHuerta et al., 2012; Eiser, Eiser, \& Stride, 2005; Grau, \& Espada, 2012; López, \& Chacón, 1999), destacando la diversidad de redes sociales que se activan, los múltiples tipos de apoyo que brindan, así como los efectos de éstas en las familias.
Cualquier proceso de enfermedad requiere de una serie de adaptaciones para las familias, sobre todo aquellas como el cáncer, donde la incertidumbre, lo prolongado del tratamiento y los costos entre otras cosas, enfrentan a éstas a cambios drásticos en la rutina, hábitos, incluso en modificaciones físicas de la vivienda. Entre los principales ajustes que mencionan las familias se encuentran los cambios en la crianza y educación de los hijos, en la organización para el cuidado del menor enfermo, en los hábitos alimenticios, en la dinámica familiar o cambio de roles entre los integrantes para cooperar en las diferentes actividades; ajustes en las rutinas escolares y laborales; ajustes económicos y sobre todo emocionales.

A lo largo de las entrevistas, las familias hablaron de diferentes grupos de personas de las que percibieron apoyo para atravesar el proceso de enfermedad de los menores. Por un lado, identificaron redes con las que ya contaban y que, dado el proceso de enfermedad, se activaron; y, por otro lado, reconocieron nuevas redes que surgieron a partir de la situación que se atravesaba. Identificaron ocho grupos distintos.

En términos generales, las redes con las que ya tenían un vínculo establecido se encuentra tanto en la familia nuclear como la extensa, los amigos y vecinos, los compañeros y maestros de la escuela, los compañeros del trabajo y los grupos religiosos. Y los tres grupos restantes son las nuevas redes de apoyo que incluyen a los padres de otros niños hospitalizados, el personal del hospital y las fundaciones. 
Aunque existe una amplia clasificación de los tipos de apoyo que se pueden recibir a través de las redes sociales, López y Chacón (1999), hacen referencia a tres tipos: emocional, tangible o instrumental y el informacional. Las familias entrevistadas hablaron del apoyo emocional entremezclando las redes que lo brindaron en diferentes momentos y maneras, con elementos que reflejan su sentir sobre el ser amado, la intimidad, la confianza que se genera en otros, y, en especial, la disponibilidad de alguien con quien poder hablar, compartir sentimientos, pensamientos y experiencias. Se brinda soporte desde el momento del diagnóstico, a lo largo de todo el proceso e incluso posterior al fallecimiento de algunos pacientes.

En cuanto al apoyo informacional, este tipo de ayuda implicó proporcionar información sobre la enfermedad, tratamientos alternativos y cuestiones prácticas para la atención de los requerimientos físicos del tratamiento. Las familias entrevistadas hablaron de lo que vivieron y de las maneras como fueron adaptándose, tomando en consideración las sugerencias.

El proceso de enfermedad enfrenta a las familias con situaciones fuera de la rutina cotidiana, ante las cuales requerirán apoyo de personas tanto de los contextos inmediatos como la casa, la escuela y el trabajo, así como en el ámbito del hospital. El apoyo tangible o instrumental recibido, representa un gran alivio en cuestiones que pueden llegar a ser muy importantes para la tranquilidad de la familia, para que puedan satisfacer necesidades básicas y para que puedan solucionar diversas cuestiones necesarias en $\mathrm{su}$ vida cotidiana. Las familias hablaron de recibir soporte relacionado con medicamentos, material escolar, comida, ropa, servicio de internet, cambio de horarios y donación de sangre, entre otros.

Si bien se conocen los diversos tipos de redes y apoyos que se ofrecen, en las entrevistas se puede percibir que la experiencia va más allá de una mera clasificación de tipos de ayuda y de quienes la brindan. El vínculo entre las familias $\mathrm{y}$ sus redes de apoyo, se hace patente en el discurso expresado.

A continuación, presentamos algunas vivencias que destacan esa relación que se construye entre las familias y las redes sociales durante el proceso de la enfermedad. A través de estas narraciones se pretende tener nueva aproximación a las experiencias desde la poesía social. Lo que significa, mostrar las escenas de las relaciones que se establecen entre ellos y presentar nuestra lectura como testigos externos a partir de la interacción que establecemos con ellas y que presentamos como lecciones de humanidad. Como lo expresan Deissler y McNamee (2013):

... nuestra convicción de que la poesía social halla su realización en el momento en que dos o más seres humanos se encuentran, entablan relaciones, y comienzan a explorarse, comprenderse, y descubrirse mutuamente al hablarse y comunicarse. Cuando las personas se relacionan, recrean en su hablar algo parcialmente nuevo y común, y cada uno de los participantes 
sale de la conversación diferente de cómo entró (p. 7).

Aunque cada caso fue único, en conjunto se puede apreciar la existencia de una gran variedad de redes, que entran a dar soporte en diversas situaciones, contribuyendo de maneras diferentes durante el proceso de la enfermedad. Todos los apoyos fueron valiosos independientemente de la cantidad o frecuencia, esto es lo que nos hace apreciar cómo cada familia resalta detalles que hacen de cada una algo extraordinario.

\section{Lecciones de la familia de Andrés}

\section{De amor y hermandad}

"A ellas les estoy muy agradecida, porque son mis niñas que me han apoyado, sin ellas sería muy difícil, sin su cooperación... Ellas también lo sufren en las noches, cuando Andrés tiene evacuaciones o vómito, ellas son las que me ayudan. Se levantan a barrer, a limpiar... Darle las gracias a mis niñas y a mi hijo, ha sido muy valiente... y darle gracias a Dios que las tengo y pedirle que me de fuerzas, así como a ellos" (Mamá de Andrés, 17 de julio de 2015).

Mirar la singularidad de esta pequeña porción de la historia de la familia de Andrés, nos hace apreciar cómo una situación tan difícil como el cáncer permite que en esta familia aflore el amor, transformado sus relaciones donde las hijas más que exigir cuidado, se convierten en aliadas de colaboración con la madre, para afrontar las demandas que la situación impone.

Como adolescentes, Adriana (14 años) y Mariana (12 años) contradicen la muy extendida idea de la adolescencia como un tiempo difícil, en el que la búsqueda de la individuación y la autonomía pueden generar comportamientos rebeldes, conflictos familiares y egocentrismo, entre otras; (Nucci, Hasebe, \& Lins-Dayee, 2005; Larson, Moneta, Richards, \& Wilson, 2002 citados en Papalia, Wenkods, \& Duskin, 2010); ellas dan muestras de hermandad al cuidar y acompañar a su hermano en momentos críticos.

\section{De espiritualidad y entrega}

En especial, la familia de Andrés expresó con gran énfasis el apoyo recibido por las familias de su grupo religioso, si habláramos de los tipos de apoyos, ellos cubrirían todos. El comentario que presentamos es una muestra de ello y nos hace pensar en la espiritualidad y entrega que demuestran estas personas.

"De todo se hizo cargo una familia, una hermana testigo de Jehová se encargó de ver que ellas vayan a la escuela, que coman, que cenen, sí. Vino a quedarse aquí con sus dos niñas. Eso me dejó tranquila porque sí me estresaba con quién se iban a quedar, cómo le iban a hacer porque son días de escuela" ... "No es mi familia de sangre, pero sí es mi familia espiritual" (Mamá de Andrés, 17 de julio de 2015).

En general, consideramos que la formación espiritual muchas veces se queda solo en el discurso, estas personas dan muestra patente de la puesta en práctica de sus preceptos, transformando las relaciones en vínculos verdaderamente cercanos, incluso hasta ser considerados como parte de la familia. 


\section{De profesionalismo}

Es evidente que las familias en el hospital están en un contexto ajeno, recibiendo apoyo de familiares y grupos cercanos. Sin embargo, considerando que el personal médico es un factor clave del proceso de enfermedad, que la familia pueda sentirlos cercanos y cálidos en su figura profesional, representa para nosotras la importancia de mostrar sensibilidad, atención, compromiso, en resumen, profesionalismo al hacer su trabajo.

La atención médica, bendito Dios, las enfermeras, los enfermeros, cada cambio de turno, he visto que dan un buen servicio con los niños; aunque sean muchos cuando le toque su turno lo atienden como tiene que ser; un trato humano, porque yo veo como el doctor cuando lo pasan a checar, se preocupa verdaderamente, te hace las preguntas y lo checan; su oncólogo preguntaba por él, se preocupaba, hasta altas horas de la noche, y no sólo por él, sino por todos sus pacientes. Se ve que se preocupa y sí, no solo pasaba, leía todo el informe y cuando no estaba porque salí, él regresaba para darme la información" (Mamá de Andrés, 7 de julio de 2015).

Este comentario de Alma enfatiza detalles muy puntuales sobre la atención y el valor que para ella tiene el trato que se convierte en individualizado, a pesar de la gran cantidad de pacientes; y nos hace pensar en el profesional desde una perspectiva colaborativa-dialógica, en la que se resalta que los seres humanos somos contiguos, por lo que es fundamental mostrar un interés genuino en el paciente, así como nuestro compromiso con ellos (Anderson, \& Gehart, 2007).

En esta misma escena que nos presenta Alma, hay un elemento significativo que vale la pena resaltar, vinculado a la concepción de Anderson y Gehart (2007) del paciente como experto, que transforma la relación profesional-paciente, donde el doctor reconoce la sabiduría de Alma como mamá de Andrés y se deja guiar por ella. "Entonces me preguntaba cómo lo estoy viendo, según yo que lo conozco, si lo estoy viendo mejor, lo estoy viendo peor, y yo de esa forma veo que él se preocupaba" (Mamá de Andrés, 7 de julio de 2015).

\section{Lecciones de la familia de Pedro \\ De ética}

Otro elemento que surge de la lectura de las historias, está relacionado con lo que entendemos por ética. En el ámbito profesional se han desarrollado códigos y estándares que establecen pautas de relación con quien solicita tus servicios, como una mera relación profesional que no trasciende al ámbito cotidiano. Sin embargo, para nosotros y de acuerdo a Anderson, (2001), la ética es parte de cómo pensamos y actuamos en todos los aspectos de nuestra vida, no podemos separar artificialmente la ética de nuestra vida profesional y personal. Entiende la ética como algo que hacemos juntos, una actividad comunal, local y mutuamente determinada por las personas involucradas, específica a los participantes, situación y circunstancias; por lo 
tanto, algunas de ellas retarán el contexto ético $\mathrm{y}$ viceversa.

Esto se ve reflejado en uno de los comentarios de Esmeralda sobre la disposición de la dentista de Pedro, que traspasando la barrera de la relación profesional se ofrece a ser parte de los cuidadores en el hospital. Esto que podría parecer un ofrecimiento insignificante, nos hace pensar en la responsabilidad relacional de la que hablan Gergen y McNamee (en Swin, St George, \& Wulff, 2001) para asegurar y mantener una oportunidad de ser ético; se debe estar continuamente abierto a revisar y cuestionar cada preocupación comunal visible con nuestros clientes, colegas, comunidades profesionales, sociales y nosotros mismos. Lo que hace significativo este ofrecimiento, es que Esmeralda lo retoma como algo valioso, independientemente de si la ayuda se dio o no, lo importante es saber que contaba con alguien que no imaginaba podría involucrarse a un nivel más personal e íntimo. Esto es lo que Esmeralda menciona: "Pues... para mí era una satisfacción ver que todos lo querían... Los vecinos, la dentista, de donde lo llevaba en la esquina. Igual, hasta ella se ofreció: si no puedes quedarte en el hospital, cuando quieras yo me quedo" (Mamá de Pedro, 8 de junio de 2015).

Otra muestra de ética que nos presenta Esmeralda la podemos palpar en el ámbito laboral.

"Me apoyaron, me pagaron, todo, normal. Gracias a Dios tuve el apoyo de la empresa... A mí en el trabajo siempre me apoyaban, me apoyaban mis compañeros, mis jefes. Siempre tuve el apoyo, entonces yo trataba de acomodarme a los horarios" (Mamá de Pedro, el 8 de junio de 2015).

Este comentario nos hace recordar las ideas de S. Swim, S. St. George y D, Wulff (2001) sobre los procesos éticos, que, aunque ellos los describen en el ámbito terapéutico, a nosotros parecen oportunos para las relaciones humanas en general y específicamente en el ámbito laboral. Ellos hablan de los procesos éticos como aquellos que se construyen en las relaciones cuando los participantes definen una sociedad ética juntos y deciden las acciones; éstos son creados en la relación inmediata y consideran a las personas como dignas del más alto respeto y reverencia.

Para nosotros, esto significa que Esmeralda en su contexto de trabajo es apreciada más que como una simple empleada en términos de producción y ganancia económica, es valorada como un ser humano que merece consideración, apoyo y estima.

\section{Lecciones de vecindad}

Lo que puede representar ser vecino en una ciudad como Mérida, Yucatán en la época actual, implica una cercanía geográfica que deja abierta la opción de convivencia-distancia con las personas de tu entorno; es una coincidencia física que, aunque no te asegura identificación con el otro, te posiciona ante la posibilidad de relacionarte con los residentes de tu vecindario, para convertirte o no en alguien al que se puede acudir de manera inmediata ante una emergencia. 
Jesús hace referencia a lo significativo que fue contar con un vecino cuando él lo necesitaba, y sin tener la seguridad de que así fuera, se vuelve valiosa la ayuda porque no hay garantía de encontrarla.

De hecho en una ocasión que le habían aplicado el medicamento, lo tuvimos que llevar directamente al hospital. Y de aquí que te llames un taxi, yo lo que busco es inmediato, ¿me explico? Vecino, por favor, necesito esto. Sí, me llevaron (Papá de Pedro, el 8 de junio de 2015).

\section{Lecciones de la familia de Sofía}

\section{De intimidad}

Como parte del proceso de enfermedad de estas familias, llega un momento en el que se ha hace indispensable la donación de sangre, como lo menciona Arcelia:

Lo que pasa es que hay que estar donando. De hecho, le ponen la sangre y yo la devuelvo, pero se me hace un poco difícil porque yo no tengo muchos familiares que puedan donar y sí se me hace muy difícil devolver la sangre (Mamá de Sofía, 3 junio de 2015).

Esta situación merece dimensionarse más allá de un mero servicio a la comunidad. La donación de sangre como servicio social, implica que personas distantes se vuelven parte de las relaciones de ayuda enmarcadas en un proceso voluntario y desinteresado, ahí reside parte de su gran valor. Pero, además, queremos destacar lo que para nosotros representa "dar sangre". Socialmente dar sangre puede simbolizar compartir "linaje", origen, distinción familiar; también puede representar, en procesos de cáncer como la leucemia, dar salud, dar posibilidades de vida. Y relacionalmente, puede implicar compartirte como persona, compartir la profunda intimidad de tu cuerpo, dar una parte de tu cuerpo a otro.

\section{Lecciones de la familia de Sabina \\ De aprecio y reconocimiento}

En el transcurso de la enfermedad las familias van aprendiendo que pueden encontrarse con dos tipos de desenlace: la recuperación del menor o como en el caso de la familia de Sabina, su fallecimiento. Ante esta situación lo esperado es que las personas expresen sus condolencias, lo cual ya por sí mismo representa un apoyo emocional importante para las familias, sin embargo, hay otras demostraciones que se vuelven más significativas al dar un lugar especial a la persona, en contextos donde estas muestras de aprecio no suelen ser habituales, de ahí que nos llame la atención lo que expresan los abuelos de Sabina:

Pero el día de su velorio, fueron niños a llorar... niños que vivía lejos fueron con sus flores, y luego cuando la incineraron, ya nada más fueron dos o tres, o sea que dejó buenos recuerdos... el día de fin de curso le hicieron un homenaje en la escuela, ahora sí que post mórtem (Abuelos de Sabina, 27 julio de 2015).

Que la escuela dedique tiempo para un homenaje representa para nosotros muestra de sensibilidad humana por parte de los directivos 
de la escuela, que lejos de ver una matrícula más ven a Sabina como parte de ellos, en su relación con los niños, la escuela y en general la comunidad educativa; siendo esto también una lección para todos como compañeros y amigos del valor de las personas a través del recuerdo.

En este sentido, si retomamos el origen de la palabra recordar, viene del latín "recordari", formado de "re" (de nuevo) y "cordis" (corazón), recordar quiere decir mucho más que tener presente a alguien en la memoria, significa "volver a pasar por el corazón".

\section{De complicidad y unión}

Nosotros éramos del grupo, como quince personas que nos conocíamos, de alguna manera se vive en el hospital, te quedas tres, cuatro días, a veces una semana y media ingresado y estabas con ese grupo de tres o cuatro personas. La idea era, hacer una convivencia para que las personas que estamos padeciendo esos problemas no se sintieran tan mal (Papá de Sabina, 27 julio de 2015).

La mayoría de las veces pasan desapercibidas estas estancias prolongadas que tienen las familias en el hospital. Las salas, los pasillos y los pabellones donde se encuentran con las otras familias, se convierten, si no en su casa, sí en los espacios donde pasan largas jornadas y donde comparten dudas, miedos y saberes; que a la larga se convierten en una especie de complicidad. Es justamente esto, lo que le da gran valor a esta red de apoyo, ya que nadie como este grupo puede entender verdaderamente lo que están viviendo. Juntos atraviesan este proceso de enfermedad, con un sentido de unión y comunidad.

\section{Discusión}

Aproximarte a las vivencias de las personas desde su propia perspectiva, permite mirar de manera más palpable esas sutilezas de la experiencia humana, favoreciendo el aprecio y la valoración de lo que es propio de situaciones tan críticas y dolorosas como las enfermedades crónicas, entre las que se encuentra el cáncer.

Las ironías de la vida muchas veces sitúan a las familias en contextos de dolor y colaboración. Es admirable que situaciones extremas promueven una profunda solidaridad entre las familias, que surge espontáneamente del dolor. Esta es una clara muestra de humanidad, de la capacidad que tenemos de empatizar y dar a los demás cuando nos encontramos en situaciones similares de crisis.

Por otra parte, cuando somos observadores del dolor ajeno, muchas veces en lo cotidiano solemos mostrar cierta disposición de ayuda, pero cuando realmente nos vinculamos con otras familias, ya sea vecinos, conocidos o simplemente personas que sufren, es cuando ponemos en práctica nuestra capacidad humana de ser sensibles, generosos y proactivos en beneficio de los demás.

Mirar las redes de apoyo desde la poesía social, ha sido estar dispuestos a dejarnos impresionar y emocionar por las palabras de estas familias, lo que nos ha hecho valorar los detalles de su vida cotidiana en situaciones tan críticas, y que son justamente estas sutilezas que las familias eligen compartir lo que vuelve significativas a las redes de apoyo. Es innegable valor de ellas 
dada la cantidad y tipo de ayuda brindada, pero el valor más profundo para nosotros reside en la relación que se construye entre personas en ese extraordinario encuentro humano.

\section{Referencias}

Amezcua, M. (2000). El trabajo de campo etnográfico en salud. Una aproximación a la observación participante. Index de Enfermería, (30), 30-35 Recuperado de http://www. index-f.com/cuali/observación.pdf

Anderson, H. (2001). Ethics and Uncertainty: Brief unfunished thoughts. Journal of Systemic Therapies, 20, 3-6.

Anderson, H., \& Gehart, D. (2007). Collaborative Therapy, Relationships and Conversations that Make a Difference. New York: Routledge.

Ávila, R. K. (2016). La experiencia de familias con hijos hospitalizados por cáncer y los ajustes a su situación (Tesis de maestría). México: Universidad Autónoma de Yucatán.

Barreto, P., \& Saiz, M. C. (2007). Muerte y duelo. Madrid: Síntesis.

Castañeda, J. (2006). El apoyo social como amortiguador de la desesperanza en padres de niños hospitalizados. Psicología Iberoamericana, 14(2), 36-45.

Deissler, K., \& McNamee, S. (2013). La poesía social de la conversación terapéutica. Inventarse a sí mismo mediante una reflexión comprometida con la relación. Ohio, EEUU: Taos Institute Publications/WorldShare Books.
Eiser, C., Eiser, R., \& Stride, C. (2005). Quality of life in children newly diagnosed with cancer and their mothers. Biomed Central, 3(1), 3-29. Recuperado de http://www.ncbi. nlm.nih.gov/pmc/articles/PMC1097748/ pdf/1477-7525-3-29.pdf

Frankl, V. (1973). El hombre en busca de sentido. Barcelona: Herder.

García, R., \& Day, C. (2009). Family Partnership Model As A Framework To Address Psychosocial Needs In Pediatric Cancer Patients. Psicooncología, 6(2-3), 357-372. Recuperado de: http://revistas. ucm.es/index.php/PSIC/article/view/ PSIC0909220357A/15151.

García, R. (2005). Hospitalización de niños y adolescentes. Revista de Medicina Clínica, 14(4), 236-241.

Gergen, K., \& Gergen, M. (2013). Reflexiones sobre la construcción social. México: Paidós. Grau, C., \& Espada, M. C. (2012). Percepciones de los padres de niños enfermos de cáncer sobre los cambios en las relaciones familiares. Psicooncología, 9(1), 125-136. Recuperado de http://revistas.ucm.es/index.php/PSIC/ article/view/39142/37747.

Krippendorff, K. (1990). Metodología de análisis de contenido: Teoría y práctica. México: Paidós Comunicación.

López-Cabanas, M., \& Chacón, F. (1999). Intervención psicosocial y servicios sociales. Madrid: Síntesis.

López-Huerta, J. A., Álvarez-Bermúdez, J., \& González-Romo, R. A. (2012). La experiencia 
psicosocial de padres de hijos con leucemia en un hospital público de la ciudad de San Luis Potosí. Psicooncología, 9(1), 137-150. Recuperado de http://revistas.ucm.es/index. php/PSIC/article/view/39143/37748.

Papalia, D., Wendkos, S., \& Duskin, R. (2010). Desarrollo Humano. México: McGrawHill. Rosemblatt, P. (1999). The social context for private feelings. En M. Stroebe, W. Stroebe \& R. Hansson (eds). Handbook of Berevament. Theory, Research and Intervention ( $\mathrm{pp}$. 255-267). London-New York: Cambridge University Press.

Swin, S, St George, S., \& Wulff, D. (2001). Process ethics: A collaborative partnership. Journal of Systemic Therapies, 20, 12-24.

Vieytes, R. (2009). Investigación cualitativa en Ciencias Sociales. Temas problemas y aplicaciones. Buenos Aires: Language Learning.

Recibido: 17/05/2018

Revisado: 18/06/2018

Aceptado: 21/07/2018 